\title{
Time for Progress
}

Epidemiology as well as management of tumours of the upper digestive tract have dramatically changed during the last 10 years: the incidence of adenocarcinomas of the esophagogastric junction has increased significantly, however, at the same time the power and quality of the available diagnostic tools experienced a substantial amelioration. With the rapid progress in molecular biology, the molecular mechanisms of tumourigenesis became more central to the oncologists' understanding of malignant diseases. These changes result in modification of the therapeutic approach. While in the past treatment of malignancies of the upper digestive tract was mainly based on simple resection of the malignant process, there is now not only a growing acceptance but even a frank preference of multimodal therapeutic concepts.

The aim of the focus in this issue of ONKOLOGIE is a critical analysis of the current knowledge of molecular mechanisms as well as of the present situation in clinical management of upper digestive tract malignancies.

The article of Sutter et al. [1] describes molecular mechanisms of tumourigenesis. The manipulation of these mechanisms is already used in the treatment of malignant disease and they represent a potential basis for further innovative therapeutic interventions. The principle of specific targeting of previously identified molecules essential for tumourigenesis or tumour cell survival has left the experimental stage and now shows impressive clinical success, e.g. in gastrointestinal stromal tumours. This is an important step towards an individually tailored therapy adapted to the specific situation of the patient. A further important prerequisite for an individualised therapy is the constant improvement of diagnostic procedures permitting the precise assessment of tumour stage and tumour dissemination.

New imaging methods transcending classical high resolution CT/MRT techniques are presented in the article of Gretschel et al. [2]. Positron emission tomography is a first step towards a functional imaging. These techniques are precursors of different methods for molecular imaging which will assess information on tumour localisation and extension as well as on the molecular characteristics and metabolic activity of the tumour tissue. Today, the importance of these techniques to guide the physician in the decision process concerning therapeutic strategies becomes increasingly clear.

Multimodal therapy is always a multidisciplinary approach. Its importance and potentials from a medical oncologists' point of view are described by Stahl [3], and Fietkau [4] presents the view of a specialist in radiooncology. The comparison of both articles illustrates the 'discipline-related' differences in evaluation and interpretation of results of published clinical trials. Even if there is no universal therapeutic concept for the different tumour situations, the two articles show the direction of potential developments of the multimodal therapy complementing the surgical treatment. It will be indispensable to assess these multimodal therapeutic concepts in thoroughly planned, randomised clinical trials on sufficiently large patient groups.

This also applies to the new therapeutic approaches introduced by Menges and Hoehler [5] in the concluding article. The emerging perspectives for treatment optimisation are impressive and should stimulate all our efforts to further ameliorate the therapeutic concepts for patients with these tumour entities. The current issue of ONKOLOGIE seeks to divulge the available data on multimodal therapy of malignancies of the upper digestive tract in order to stimulate the interdisciplinary cooperation for further improvement of these innovative strategies. New therapeutic concepts for the treatment of upper gastrointestinal tract malignancies are urgently needed!

Guido Adler, Ulm Peter M. Schlag, Berlin

\section{References}

1 Sutter AP, Zeitz M, Scherübl H: Recent results in understanding molecular pathways in the medical treatment of esophageal and gastric cancer. Onkologie 2004;27:17-21.

2 Gretschel S, Moesta KT, Hünerbein M, Lange T, Gebauer B, Stroszczinski C, Bembenek A, Schlag PM: New concepts of staging in gastrointestinal tumors as a basis of diagnosis and multimodal therapy. Onkologie 2004;27:23-30.

3 Stahl M: Adjuvant chemoradiotherapy in gastric cancer and carcinoma of the oesophago-gastric junction. Onkologie 2004;27:33-36.

4 Fietkau R: Definitive and neoadjuvant radiochemotherapy for squamous cell carcinoma of the esophagus. Onkologie 2004;27:39-44.

5 Menges M, Hoehler T: Innovative drugs and strategies in medical treatment of upper gastrointestinal tract carcinomas. Onkologie 2004;27:47-53

\begin{tabular}{ll}
\hline KARGER & @ 2004 S. Karger GmbH, Freiburg \\
Fax +49 7614520714 & Accessible online at: \\
$\begin{array}{l}\text { E-mail Information@Karger.de } \\
\text { www.karger.com }\end{array}$ & www.karger.com/onk
\end{tabular}

Prof. Dr. Dr. h.c. Peter M. Schlag

Universitätsmedizin Charité, Campus Berlin Buch

Robert-Rössle-Klinik im HELIOS Klinikum Berlin

Lindenberger Weg 80, D-13125 Berlin

Tel. +49 30 9417-1400, Fax -1404

E-mail schlag@rrk.charite-buch.de 